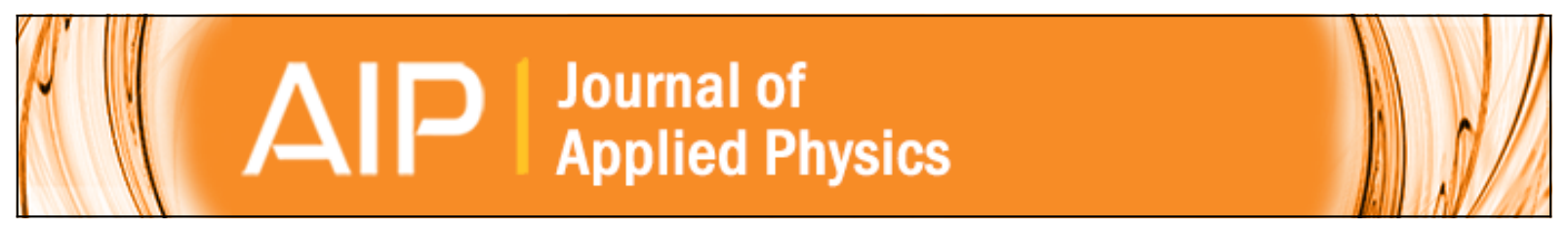

The effect of matrix and substrate on the coercivity and blocking temperature of selfassembled Ni nanoparticles

Jeremiah T. Abiade, Sang Ho Oh, Dhananjay Kumar, Maria Varela, Stephen Pennycook, Haizhong Guo, Arunava Gupta, and Jagannathan Sankar

Citation: Journal of Applied Physics 104, 073910 (2008); doi: 10.1063/1.2992528

View online: http://dx.doi.org/10.1063/1.2992528

View Table of Contents: http://scitation.aip.org/content/aip/journal/jap/104/7?ver=pdfcov

Published by the AIP Publishing

Articles you may be interested in

Magnetoresistive effects in Co/Pd multilayers on self-assembled nanoparticles (invited)

J. Appl. Phys. 107, 09C506 (2010); 10.1063/1.3350909

Synthesis of self-assembled monodisperse $3 \mathrm{~nm}$ FePd nanoparticles: Phase transition, magnetic study, and surface effect

J. Appl. Phys. 104, 123906 (2008); 10.1063/1.3042259

Temperature dependence and magnetocrystalline anisotropy studies of self-assembled L $10-\mathrm{Fe} 55 \mathrm{Pt} 45$ ferromagnetic nanocrystals

J. Appl. Phys. 101, 123918 (2007); 10.1063/1.2747209

Coercivity and remanence in self-assembled FePt nanoparticle arrays

J. Appl. Phys. 93, 7041 (2003); 10.1063/1.1557398

Magnetic properties of self-assembled nanoscale La $2 / 3 \mathrm{Ca} 1 / 3 \mathrm{MnO} 3$ particles in an alumina matrix Appl. Phys. Lett. 79, 1327 (2001); 10.1063/1.1399001

MIT LINCOLN LABORATORY CAREERS

Discover the satisfaction of innovation and service to the nation
- Space Control

- Air \& Missile Defense

- Communications Systems \& Cyber Security

- Intelligence, Surveillance and

Reconnaissance Systems

\section{LINCOLN LABORATORY}

MAsSachusetts InStitute of TeChNOLOGY

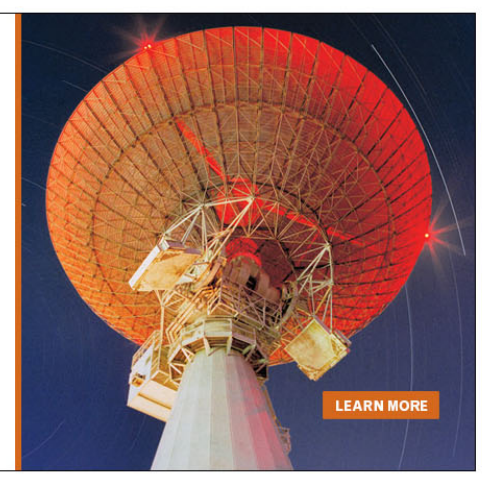




\title{
The effect of matrix and substrate on the coercivity and blocking temperature of self-assembled Ni nanoparticles
}

\author{
Jeremiah T. Abiade, ${ }^{1, a)}$ Sang Ho $\mathrm{Oh}^{2}{ }^{2}$ Dhananjay Kumar, ${ }^{3,4}$ Maria Varela, ${ }^{4}$ \\ Stephen Pennycook, ${ }^{4}$ Haizhong Guo, ${ }^{5}$ Arunava Gupta, ${ }^{5}$ and Jagannathan Sankar ${ }^{3}$ \\ ${ }^{1}$ Laboratory for Oxide Research and Education (L.O.R.E.), Department of Materials Science and Engineering \\ and Department of Mechanical Engineering, Virginia Polytechnic Institute and State University, \\ Blacksburg, Virginia 24061, USA \\ ${ }^{2}$ Division of Electron Microscopic Research, Korea Basic Science Institute, Eoeun-dong 52, Daejeon $305-$ \\ 333, Korea \\ ${ }^{3}$ Department of Mechanical and Chemical Engineering and Center for Advanced Materials and Smart \\ Structures (CAMSS), North Carolina A\&T State University, Greensboro, North Carolina 27411, USA \\ ${ }^{4}$ Condensed Matter Sciences Division, Oak Ridge National Laboratory (ORNL), Oak Ridge, Tennessee 37831, \\ USA \\ ${ }^{5}$ Departments of Chemistry and Chemical Engineering and Center for Materials for Information Technology \\ (MINT), University of Alabama, Tuscaloosa, Alabama 35487, USA
}

(Received 31 December 2007; accepted 19 August 2008; published online 15 October 2008)

We have shown that the magnetic properties of nanoparticles may be tuned from superparamagnetic to ferromagnetic by changing the substrate or thin film matrix in which they are embedded. Nickel nanoparticles were embedded into alumina, titanium nitride, and cerium oxide matrices on both silicon and sapphire substrates via pulsed laser deposition. The laser ablation time on the nickel target was kept constant. Only nickel nanoparticles in cerium oxide showed characteristics of ferromagnetism (room temperature coercivity and remanence). Ni nanoparticles, in either alumina or titanium nitride, possessed blocking temperatures below $200 \mathrm{~K}$. Detailed scanning transmission electron microscopy analysis has been conducted on the samples embedded into cerium oxide on both substrates and related to the magnetic data. () 2008 American Institute of Physics. [DOI: $10.1063 / 1.2992528$ ]

\section{INTRODUCTION}

Nanostructured magnetic materials display unique properties that are being aggressively researched for a variety of technological applications. Perhaps the best example of an emerging application is the magnetic recording industry in which storage densities $>\mathrm{Tb} / \mathrm{in}^{2}$ are being pursued. ${ }^{1-4}$ The implementation of nanoparticle based recording media is complicated by the phenomenon of superparamagnetism. Superparamagnetism is a small particle effect that occurs as the nanoparticle diameter is reduced below some critical value. ${ }^{5}$ Superparamagnetism (SPM) particles are characterized by a lack of remanence and coercivity due to the effect of thermal fluctuations on the direction of the magnetization vector. For SPM samples, a blocking temperature $\left(T_{B}\right)$ exists, such that when $T<T_{B}$ the sample will behave as a ferromagnet. For $T>T_{B}$, thermal fluctuations are sufficiently energetic to overcome the coercive field, causing random rotation of the nanoparticle spins, which make the particles unsuitable for data storage. High coercivity FePt nanoparticles have been identified as possible solutions to the superparamagnetic limit imposed on nanomagnetic media. ${ }^{2,6}$ Chemical routes have been predominately used for FePt synthesis. ${ }^{2,6,7}$ However, after synthesis, additional thermal treatments $\left(>500{ }^{\circ} \mathrm{C}\right)$ are required to achieve magnetic and crystallographic ordering of FePt into its highly anisotropic $L_{10}$ phase

\footnotetext{
${ }^{a)}$ Author to whom correspondence should be addressed. Tel.: 540-231-1469. FAX: 540-231-8919.Electronic mail: jabiade@ vt.edu.
}

that is responsible for the enhanced coercivity. ${ }^{6}$ Heating above $550{ }^{\circ} \mathrm{C}$ results in considerable particle coalescence and loss of particle positional order, ${ }^{6,7}$ which increases the switching volumes, negating the advantageous aspects of nanoparticle based media. ${ }^{6}$

Physical vapor deposition techniques such as pulsed laser deposition (PLD) may provide an alternate route to achieve monodispersed, high coercivity magnetic nanoparticles. ${ }^{8}$ The particles may be formed in the gas phase $^{9,10}$ or they may be grown directly onto thin film templates. ${ }^{11}$ For sufficiently controlled growth processes, the nanocluster size can be tailored, providing simultaneous synthesis, deagglomeration, and passivation of nanomaterials in situ. We have used PLD to deposit magnetic nanoparticles in various thin film matrices alumina $\left(\mathrm{Al}_{2} \mathrm{O}_{3}\right)$, titanium nitride (TiN), and cerium oxide $\left(\mathrm{CeO}_{2}\right)$. The crystallinity of the thin film matrix has previously been shown to significantly affect the magnetic properties of embedded nanoparticles. ${ }^{12}$ The $\mathrm{Ni}$ particles evolve as three-dimensional (3D) self-assembled clusters because of the tendency of metallic materials to follow the Volmer-Weber growth mode. ${ }^{13}$ For sufficiently short deposition times, the growing films are not continuous, having the form of discrete islands or clusters. These islands or clusters may be used to mimic the properties of nanoparticles. In this article, we will discuss the effect of thin film matrix $\left(\mathrm{Al}_{2} \mathrm{O}_{3}, \mathrm{TiN}, \mathrm{CeO}_{2}\right)$ and substrate $(\mathrm{Si}$, sapphire) on the coercivity and blocking temperature of self-assembled $\mathrm{Ni}$ nanoparticles. The Ni nanoparticle size can be controlled by tailoring the deposition time (number of laser pulses on the 

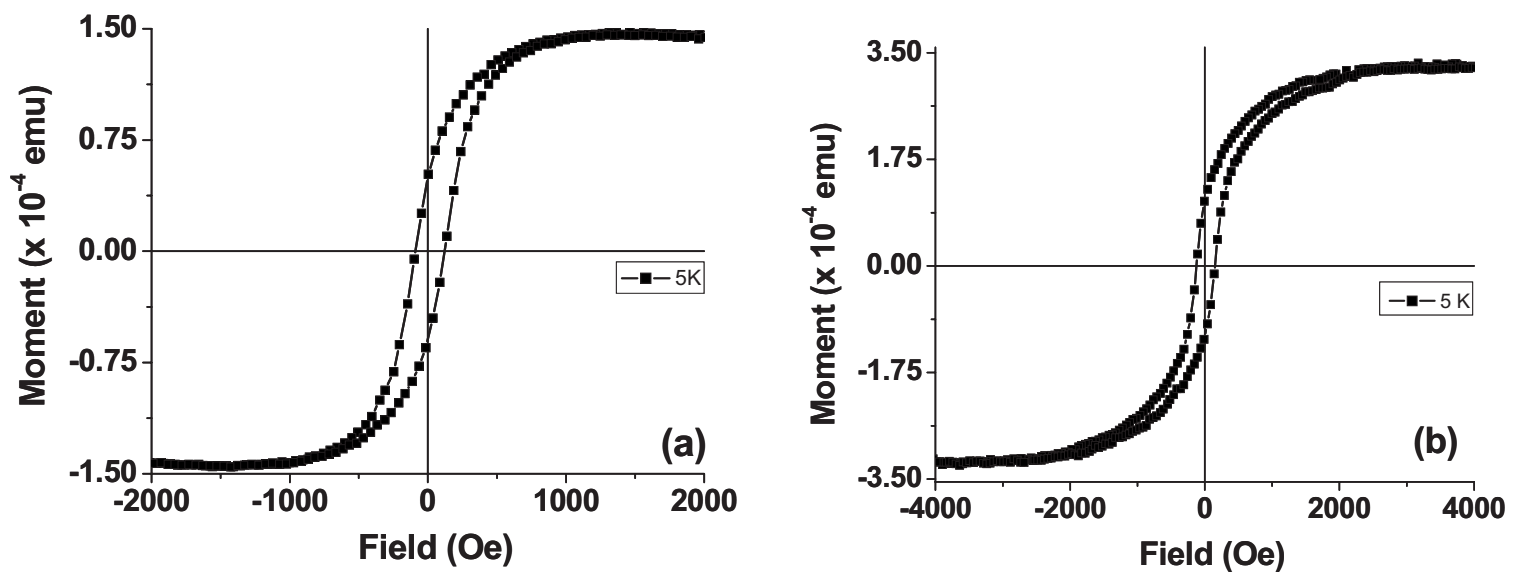

FIG. 1. Hysteresis loops recorded at $5 \mathrm{~K}$ for Ni embedded in an $\mathrm{Al}_{2} \mathrm{O}_{3}$ matrix (a) on $\mathrm{Si}$ and (b) on a sapphire substrate.

Ni target) as the films grows in the respective thin film matrix.

\section{EXPERIMENTAL}

Nickel nanoparticulates were embedded in alumina $\left(\mathrm{Al}_{2} \mathrm{O}_{3}\right)$, titanium nitride $(\mathrm{TiN})$, and cerium oxide $\left(\mathrm{CeO}_{2}\right)$ thin films on $\mathrm{Si}(100)$ and sapphire (c-plane) substrates by laser ablation during PLD. The PLD system consists of a multitarget carousel and a $\mathrm{KrF}$ excimer laser with $\lambda=248 \mathrm{~nm}$ (Lambda Physik). Prior to each deposition, the substrates were cleaned in acetone. The Si substrates were also etched in dilute hydrofluoric acid to remove the native oxide layer. After the desired chamber pressure was reached $\left(\sim 10^{-7}\right.$ torr $)$, the substrates were maintained at the deposition temperature $\left(600-650{ }^{\circ} \mathrm{C}\right)$ for $30 \mathrm{~min}$ prior to each run. All films were deposited in vacuum. The target to substrate distance was maintained at $4.8 \mathrm{~cm}$. The multilayered structure consisted of an initial thin film layer $(\sim 50 \mathrm{~nm})$ and alternating layers of nickel nanoparticles and the respective thin film spacer layers $(\sim 25 \mathrm{~nm})$. Each sample consists of five alternating nanoparticle/thin film layers. The number of pulses on the $\mathrm{Ni}$ target was constant for all samples. The nominal deposition rate of the Ni layers was $\sim 0.1 \mathrm{~nm} / \mathrm{s}$. The depositions were carried out simultaneously on $\mathrm{Si}$ and sapphire substrates.

Powder x-ray diffraction (XRD) analysis was performed using a Rigaku diffractometer with $\mathrm{Cu} K \alpha$ source. The magnetic properties of the nanocomposites were studied using a physical property measurement system equipped with the vibrating sample magnetometer (VSM) option from Quantum Design and superconducting quantum interference device (SQUID) magnetometer. The VSM and SQUID were used to study the field and temperature dependence of the magnetic moment of $\mathrm{Ni}$ particles for the various thin film matrix/ substrate combinations. For magnetization versus temperature measurements, the moment was measured in both the zero field cooled (ZFC) and field cooled (FC) modes. During the ZFC measurement, the samples were cooled to $10 \mathrm{~K}$ with no applied field. The moment was then measured while warming the samples up to $300 \mathrm{~K}$ in a magnetic field of 50 Oe. For FC measurements, the samples were cooled to $10 \mathrm{~K}$ in the respective fields and the moment was measured while warming to room temperature. The temperature dependence of the coercivity was obtained from the magnetization versus field loops obtained at different temperatures.

Cross-sectional specimens for electron microscopy analysis were prepared following conventional methods consisting of dimpling and Ar-ion milling to obtain electron transparency. Scanning transmission electron microscopy (STEM) imaging was carried out in a VG HB501UX microscope operated at $100 \mathrm{kV}$ equipped with a Nion aberration corrector. High angle annular dark-field (ADF) and brightfield (BF) images were recorded simultaneously. Spectrum images were acquired by using a Gatan Enfina electron energy loss spectroscopy (EELS) attachment on the STEM. ADF images and EELS spectra were acquired simultaneously in the energy range of 430-966.4 eV with the energy dispersion of $0.4 \mathrm{eV}$ per channel at an acquisition time of 0.5 s. Quantitative elemental mapping after background subtraction was performed using the respective core-loss edges: Ni $L_{2,3}(854 \mathrm{eV})$, Ce $M_{4,5}(883 \mathrm{eV})$, and $\mathrm{O} K(532 \mathrm{eV})$.

\section{RESULTS AND DISCUSSIONS}

The three thin film matrices $\left(\mathrm{Al}_{2} \mathrm{O}_{3}, \mathrm{CeO}_{2}\right.$, and $\left.\mathrm{TiN}\right)$ in this study have widely varying crystallographic properties depending on growth conditions and substrate. For example, $\mathrm{Al}_{2} \mathrm{O}_{3}$ films are generally amorphous regardless of deposition conditions and substrate except at higher deposition temperatures. ${ }^{14}$ Epitaxial $\mathrm{CeO}_{2}$ films have previously been obtained on both sapphire ${ }^{15}$ and silicon ${ }^{16}$ substrates whereas epitaxial TiN films grown in vacuum have been reported on silicon $^{12,17,18}$ not sapphire. The varying degrees of crystallinity of the thin film matrix permit texturing of the magnetic properties of the embedded particles. Figure 1 shows typical $M-H$ loops recorded at $5 \mathrm{~K}$ for the Ni particles embedded in an $\mathrm{Al}_{2} \mathrm{O}_{3}$ matrix on a Si substrate [Fig. 1(a)] and a sapphire substrate [Fig. 1(b)]. The coercivity $\left(H_{c}\right)$, saturation magnetization $\left(M_{s}\right)$, remanent magnetization $\left(M_{r}\right)$, and remanence ratio $\left(M_{r} / M_{s}\right)$ for the various matrix/substrate combinations measured at $5 \mathrm{~K}$ are given in Table I. In all cases, the $\mathrm{Ni}$ deposition time was kept constant. $M_{s}$ is $\sim 10^{-4} \mathrm{emu}$ for all samples. All of the $M-H$ loops become saturated at fields less than $\sim 3$ kOe except for $\mathrm{Ni}$ embedded in $\mathrm{CeO}_{2}$ on sapphire, which requires a field in excess of $5 \mathrm{kOe}$. The coercivity at 5 
TABLE I. Coercivity $\left(H_{c}\right)$, saturation magnetization $\left(M_{s}\right)$, remanent magnetization $\left(M_{r}\right)$, and remanence ratio $\left(M_{r} / M_{s}\right)$ for various matrix substrate combinations measured at $5 \mathrm{~K} .{ }^{*} H_{c}(0 \mathrm{~K})$ obtained from fit to data in Fig. 2.

\begin{tabular}{lccccc}
\hline \hline $\begin{array}{l}\text { Matrix/substrate } \\
\text { combination }\end{array}$ & $H_{c}(5 \mathrm{~K}) \mathrm{Oe}$ & ${ }^{*} H_{c}(0 \mathrm{~K}) \mathrm{Oe}$ & $M_{s}\left(10^{-4} \mathrm{emu}\right)$ & $M_{r}\left(10^{-4} \mathrm{emu}\right)$ & $M_{r} / M_{s}$ \\
\hline $\mathrm{Al}_{2} \mathrm{O}_{3} / \mathrm{Si}$ & 100 & 130 & 1.45 & 0.54 & 0.37 \\
$\mathrm{Al}_{2} \mathrm{O}_{3} / \mathrm{Al}_{2} \mathrm{O}_{3}$ & 135 & 165 & 3.30 & 1.10 & 0.33 \\
$\mathrm{TiN} / \mathrm{Si}$ & 265 & 320 & 0.92 & 0.35 & 0.38 \\
$\mathrm{TiN} / \mathrm{Al}_{2} \mathrm{O}_{3}$ & 190 & 234 & 1.30 & 0.60 & 0.46 \\
$\mathrm{CeO}_{2} / \mathrm{Si}$ & 370 & 400 & 2.34 & 1.12 & 0.48 \\
$\mathrm{CeO}_{2} / \mathrm{Al}_{2} \mathrm{O}_{3}$ & 315 & 335 & 6.00 & 1.59 & 0.27 \\
\hline \hline
\end{tabular}

$\mathrm{K}$ changes dramatically depending on the matrix/substrate combination. Generally, the coercivity is enhanced for matrix substrate combinations in which the matrix layers are more crystalline. The lowest $H_{c}$ values are observed for Ni particles embedded in an alumina matrix. $\mathrm{Ni}$ in $\mathrm{CeO}_{2}$ possessed $H_{c}$ values of 370 and $315 \mathrm{Oe}$ on $\mathrm{Si}$ and sapphire, respectively, at $5 \mathrm{~K}$. Ni particles in TiN matrices on both $\mathrm{Si}$ and sapphire possessed intermediate values between that of the alumina and $\mathrm{CeO}_{2}$ matrix (maximum in $H_{c}$ also on $\mathrm{Si}$ ). The approximate threefold increase in $H_{c}$ for $\mathrm{Ni}$ embedded in $\mathrm{CeO}_{2}$ in comparison to $\mathrm{Ni}$ embedded in $\mathrm{Al}_{2} \mathrm{O}_{3}$ is illustrative of the ability to tailor the magnetic properties. The remanence ratio allows insight into interparticle interactions. Generally, $M_{r} / M_{s} \sim 0.5$ is observed for random, noninteracting particles. As shown in Table I, only matrix/substrate combinations of TiN/sapphire and $\mathrm{CeO}_{2} / \mathrm{Si}$ are suggestive of noninteracting particles.

The Ni nanoparticles are SPM in all matrices except $\mathrm{CeO}_{2}$. Only $\mathrm{Ni}$ in $\mathrm{CeO}_{2}$ on $\mathrm{Si}$ and sapphire possesses the characteristic features of ferromagnetism (FM) such as room temperature coercivity and remanence. Figure 2 shows the linear dependence of coercivity on the square root of the temperature $\left(T^{1 / 2}\right)$ obtained from the relation $H_{c}(T)$ $=H_{c}(0 \mathrm{~K})\left[1-\left(T / T_{B}\right)^{1 / 2}\right]$, typical of nanoparticle samples. ${ }^{19}$ $H_{c}(0 \mathrm{~K})$ is the coercivity at $0 \mathrm{~K}$, which is strongly dependent on the intrinsic anisotropy of the system, $T$ is the measurement temperature, and $T_{B}$ is the blocking temperature,

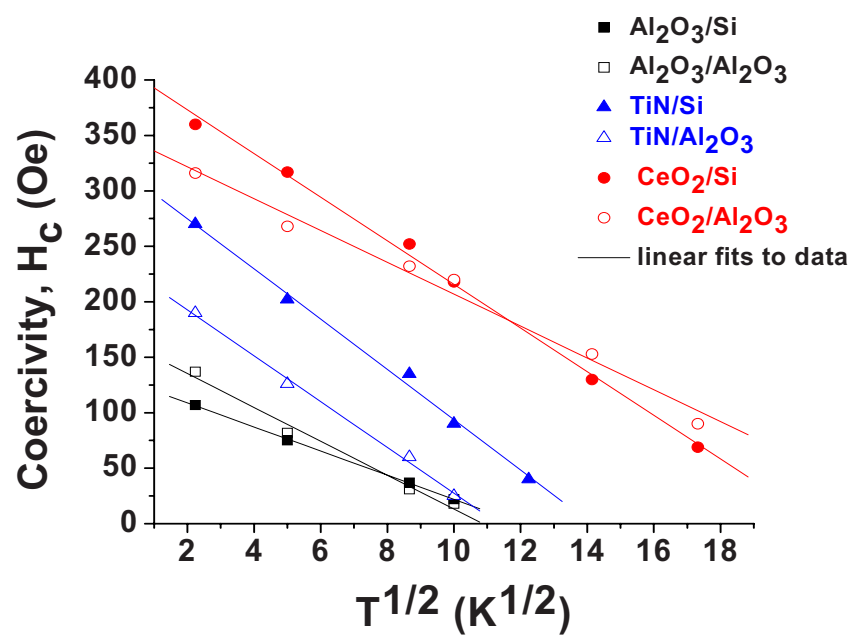

FIG. 2. (Color online) Plot of coercivity vs square root of temperature for $\mathrm{N}$ in various matrix/substrate combinations. which was previously discussed. The trend lines are linear fits to the data, which were used to obtain $H_{C}(0 \mathrm{~K})$ and $T_{B}$. The coercivity was obtained from $M-H$ loops measured at different temperatures. From the linear fits, Fig. 2 clearly shows that the $T_{B}$ for the $\mathrm{Ni}$ particles in alumina and $\mathrm{TiN}$ is well below $300 \mathrm{~K}$. For $\mathrm{Ni}$ in alumina, the estimated $T_{B}$ is $\sim 117$ and $143 \mathrm{~K}$ on Si and sapphire, respectively. Similarly, $T_{B}$ for Ni in TiN is $\sim 199 \mathrm{~K}$ on $\mathrm{Si}$ and $128 \mathrm{~K}$ on sapphire. The estimated $T_{B}$ for $\mathrm{Ni}$ in alumina and TiN agrees well with experimentally determined values represented by irreversibility in the magnetization versus temperature curves in Fig. 3. Except for the TiN sample, the trend lines for the data in Fig. 2 follow a nonmonotonic trend. For example, the curves for $\mathrm{Ni}$ in alumina and $\mathrm{Ni}$ in $\mathrm{CeO}_{2}$ on the different substrates intersect. This nonmonotonic trend in the slopes of $H_{c}$ versus $T^{1 / 2}$ may be attributed to multiple effects: distributions in particles size and/or shape, size dependence of anisotropy constants, and/or particle-particle interactions. The interparticle interactions may be enhanced for tighter packing. Uninfluenced by thermal effects, the low temperature coercivity is directly related to the nanoparticle magnetic anisotropy. Figures 1-3 and Table I clearly demonstrate the ability to texture the magnetic properties by varying the matrix and/or substrate. We will now discuss the samples with Ni particles embedded in a $\mathrm{CeO}_{2}$ matrix in greater detail.

Figure 4 shows the remanence ratio $\left(M_{r} / M_{s}\right)$ versus temperature for $\mathrm{Ni}$ embedded in $\mathrm{CeO}_{2}$ on both $\mathrm{Si}$ and sapphire substrates. The two curves show quite different temperature dependencies, which suggests the magnetic anisotropy and interparticle interactions may be different for the two samples. An increase in temperature effectively reduces the magnetic properties in part due to randomization of the interparticle interactions and degradation of the magnetic anisotropy. On the $\mathrm{Si}$ substrate, the remanence ratio changes $\sim 34 \%$ from 5 to $300 \mathrm{~K}$. The temperature dependence is much less pronounced on the sapphire substrate changing $\sim 15 \%$ from 5 to $100 \mathrm{~K}$ and remaining relatively flat up to room temperature. Several effects may contribute to the observations in Fig. 4. Generally, interparticle interactions lower the remanence, creating flux closure loops. ${ }^{20}$ Furthermore, the magnetic anisotropy is also a strong function of the nanoparticle size and temperature. For example, the cubic anisotropy constant for $\mathrm{Ni}$ decreases by a factor of 22 from low to room temperature. ${ }^{21}$ XRD analysis (not shown) indicates the $\mathrm{CeO}_{2}$ layers are highly crystalline with (111) tex- 

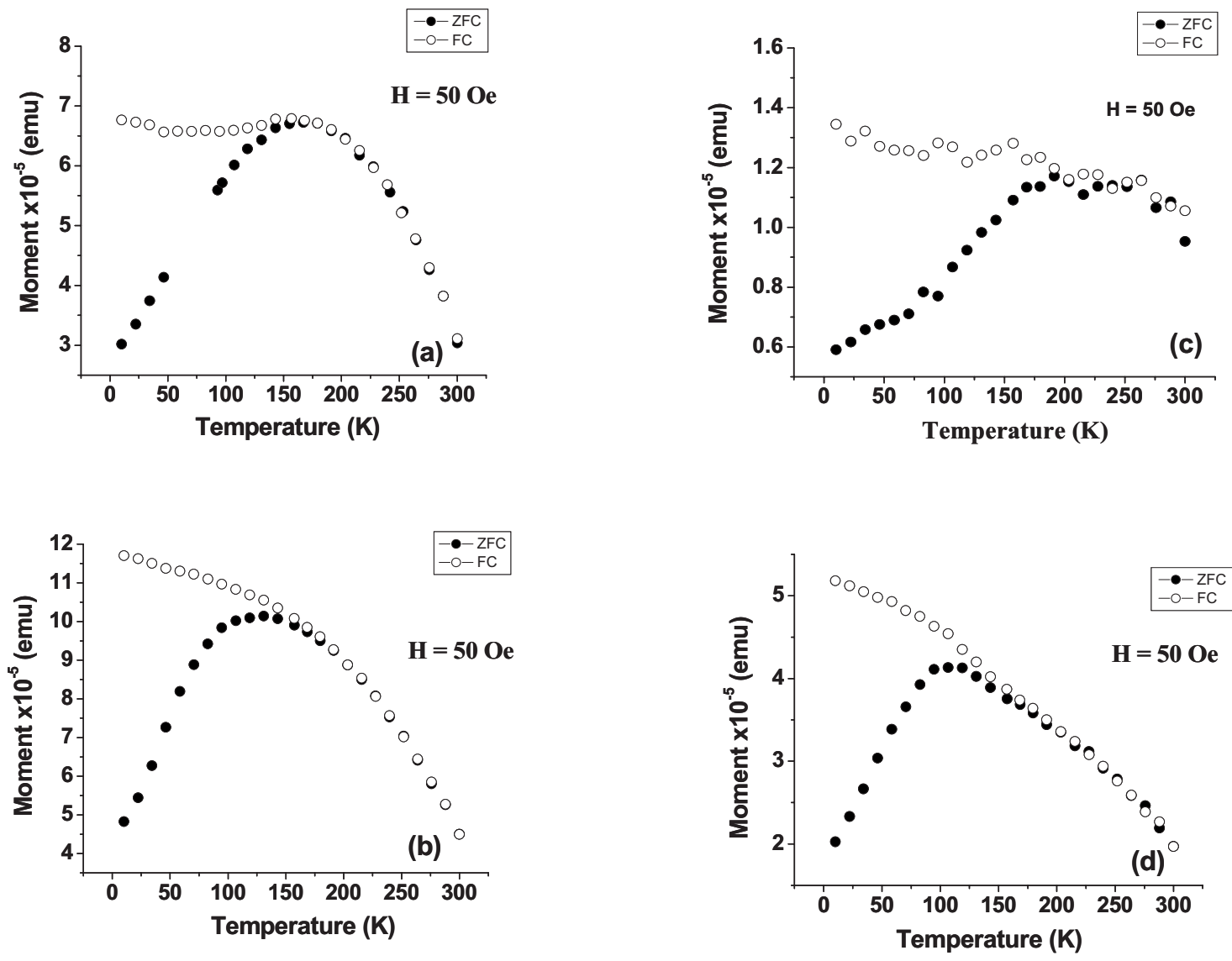

FIG. 3. Moment vs temperature measurements for $\mathrm{Ni}$ embedded in (a) $\mathrm{Al}_{2} \mathrm{O}_{3}$ matrix on $\mathrm{Si}$, (b) $\mathrm{Al}_{2} \mathrm{O}_{3}$ matrix on sapphire, (c) TiN matrix on $\mathrm{Si}$, and (d) TiN matrix on sapphire

ture on both $\mathrm{Si}$ and sapphire. Typically, the full width at half maximum of the $\mathrm{CeO}_{2}$ (111) peak is slightly lower on $\mathrm{Si}$ than on sapphire because of the similarities in crystal structure and lattice parameter. Based on our XRD data, the relative intensity of the secondary $\mathrm{CeO}_{2}$ orientations such as (200) and (220) with respect to (111) on $\mathrm{Si}$ are $10 \%$ and $<0.1 \%$, respectively. The corresponding values for (200) and (220) on sapphire are both $<0.1 \%$. In this study, the crystallinity of the $\mathrm{CeO}_{2}$ matrix layer is better on sapphire. Electron microscopy of the $\mathrm{CeO}_{2}$ layers and $\mathrm{Ni}$ particles is required for complete analysis of the magnetometry data.

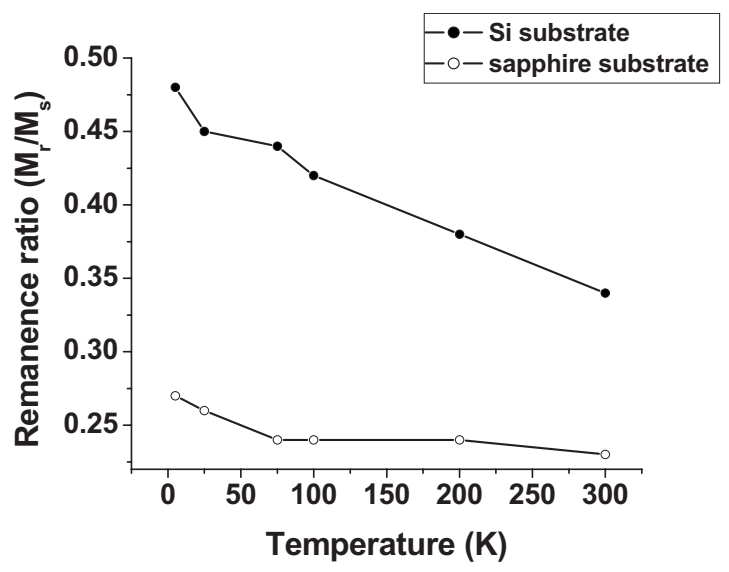

FIG. 4. Remanence ratio vs temperature for $\mathrm{Ni}$ in $\mathrm{CeO}_{2}$ on $\mathrm{Si}(\bullet)$ and sapphire $(\bigcirc)$ substrates.
Figure 5 shows a low magnification STEM image of the $\mathrm{Ni}$ particles embedded in $\mathrm{CeO}_{2}$ on $\mathrm{Si}[\mathrm{Fig}$. 5(a)] and sapphire [Fig. 5(b)] substrates. The five layers of Ni particles (bright discontinuous regions) are clearly separated by homogeneous regions of $\mathrm{CeO}_{2}$. The composite sample thickness is $\sim 230 \mathrm{~nm}$ and each layer of Ni particles is separated by 25 $\mathrm{nm} \mathrm{CeO}_{2}$ layers for both substrates. Detailed high resolution STEM imaging has been conducted on the $\mathrm{CeO}_{2}$ layers and the embedded particles. Figure 6 is a STEM image of the sample on the sapphire substrate. Figure 6(a) shows a region of the $\mathrm{CeO}_{2}$ spacer layer with a few $\mathrm{Ni}$ particles at the top of the image. Figure 6(a) also indicates that the $\mathrm{CeO}_{2} /$ sapphire interface is abrupt whereas the $\mathrm{CeO}_{2} / \mathrm{Si}$ interface (not shown) consists of native oxide $(\mathrm{Si}-\mathrm{O})$ and cerium silicate (Ce-Si-O) layers, which may explain the higher roughness and diminished crystallinity of $\mathrm{CeO}_{2}$ on $\mathrm{Si}$ versus sapphire substrates. The boxed region in Fig. 6(a) is shown at a higher magnification in Fig. 6(b). The columnar structure of the

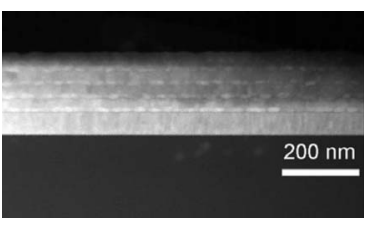

(a)

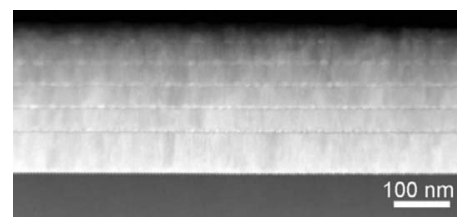

(b)
FIG. 5. ADF STEM image of five layers of Ni nanoparticles embedded in $\mathrm{CeO}_{2}$ on (a) $\mathrm{Si}$ substrate and (b) sapphire substrate. 


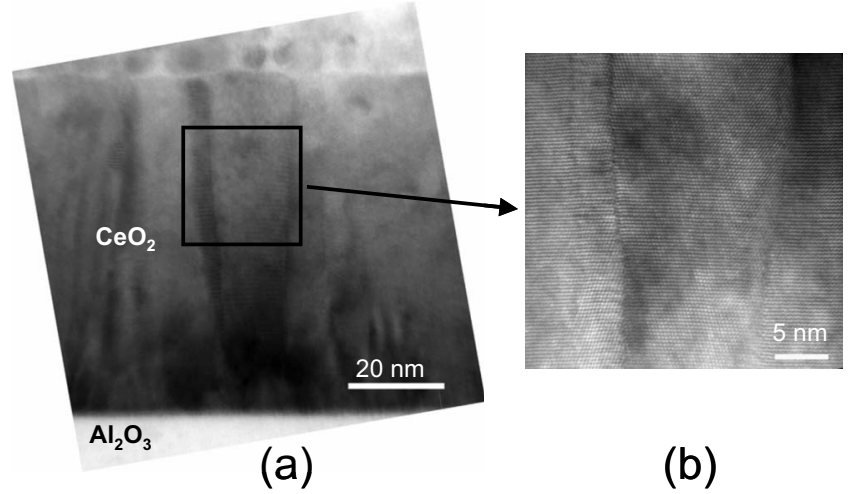

FIG. 6. BF STEM images showing morphology of $\mathrm{CeO}_{2}$ spacer layer on sapphire substrate (a) lower magnification of region in first $\mathrm{CeO}_{2}$ layer (b) higher resolution image of boxed region in (a).

$\mathrm{CeO}_{2}$ layer prominent in Fig. 6(b) was seen on both substrates. In agreement with XRD, the $\mathrm{CeO}_{2}$ layers are highly (111) oriented. The $\mathrm{CeO}_{2}$ film grown on sapphire is epitaxial (although the columnar grains are slightly misoriented by a few degrees in plane, as can be seen in Fig. 6(b)). The $\mathrm{CeO}_{2}$ film grown on the $\mathrm{Si}$ substrate also shows (111) textured orientation but its columnar grains do not possess epitaxial relationship with the substrate. This is due to the presence of a thin cerium silicate amorphous layer which results from an interfacial reaction between the growing $\mathrm{CeO}_{2}$ film and the $\mathrm{Si}$ substrate. The bulk $\mathrm{CeO}_{2}$ matrix on both $\mathrm{Si}$ and sapphire substrates is very similar. Perhaps the difference in magnetic properties may be attributed to the $\mathrm{Ni} / \mathrm{CeO}_{2}$ interface or differences in the physical characteristics (size, shape, etc.) of the Ni particles in the two samples.

Figure 7 shows high resolution STEM images of the $\mathrm{Ni} / \mathrm{CeO}_{2}$ interfaces and $\mathrm{Ni}$ particles on silicon [Fig. 7(a)] and sapphire [Fig. 7(b)] substrates. EELS (not shown), used for compositional analysis, confirmed the existence of pure metallic Ni nanoparticles on both substrates. The most striking difference in the two samples is the Ni particle size and shape. The $\mathrm{Ni}$ particles deposited in $\mathrm{CeO}_{2}$ on $\mathrm{Si}$ substrates are disk shaped with average heights of $\sim 12-14 \mathrm{~nm}$ and widths of $\sim 25 \mathrm{~nm}$ giving a width to height aspect ratio of $\sim 2$. On sapphire, the $\mathrm{Ni}$ particles are more spherical with average heights of $\sim 8 \mathrm{~nm}$ and widths of $\sim 10 \mathrm{~nm}$. Also, the $\mathrm{Ni}$ interparticle separation is larger on $\mathrm{Si}(>5 \mathrm{~nm})$ than on sapphire substrates $(\sim 1-2 \mathrm{~nm})$. The $\mathrm{Ni}$ interparticle distance increases as the number Ni layers is increased on the $\mathrm{Si}$ substrate because of the increased roughness of the $\mathrm{CeO}_{2}$

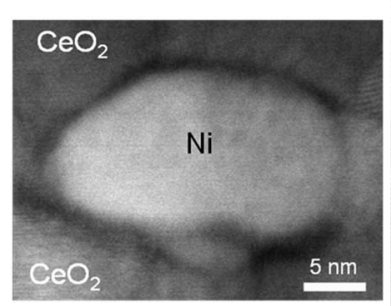

(a)

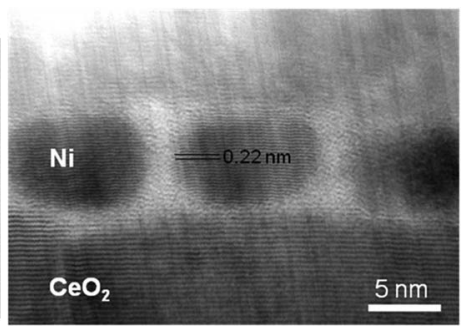

(b)
FIG. 7. BF STEM images showing $\mathrm{CeO}_{2} / \mathrm{Ni} / \mathrm{CeO}_{2}$ layers on (a) $\mathrm{Si}$ substrate and (b) sapphire substrate. buffer layers. Interparticle separation distances in excess of 3 $\mathrm{nm}$ are required to decouple the magnetic particles, ${ }^{22}$ as evidenced by the respective remanence ratios given in Table I. On the $\mathrm{Si}$ substrate, the $M_{r} / M_{s}$ value at $5 \mathrm{~K}$ is 0.48 and 0.27 on sapphire. Typically, a $M_{r} / M_{s}$ value of 0.5 is observed for random, noninteracting particles. The particles on both substrates are predominantly (111) oriented. The Ni particle interplanar spacing of $0.22 \mathrm{~nm}$ highlighted in Fig. 7(b) is close to the bulk value of 0.20 , which is indicative of $\mathrm{Ni}(111){ }^{23}$ The $\mathrm{Ni} / \mathrm{CeO}_{2}$ interface is also quite different for the two samples. On $\mathrm{Si}$ substrates, the crystalline $\mathrm{CeO}_{2}$ directly contacts $\mathrm{Ni}$ particles, establishing local epitaxy whereas the $\mathrm{CeO}_{2}$ surrounding $\mathrm{Ni}$ particles is amorphous on sapphire.

The evolution of the self-assembled Ni nanoparticles is a dynamic process dictated by thermodynamic and kinetic factors during the growth process. In the $\mathrm{Ni} / \mathrm{CeO}_{2}$ system, $\mathrm{Ni}$ evolves as 3D clusters or nanoparticles because the Ni surface free energy $\left[\sim 2.0\right.$ versus $1.06 \mathrm{~J} / \mathrm{m}^{2}$ for $\mathrm{Ni}$ (111) (Ref. 18) and $\mathrm{CeO}_{2}$ (111) (Ref. 24), respectively] is much larger than that of $\mathrm{CeO}_{2}$ (i.e., Volmer-Weber growth). This phenomenon is commonly observed for metal on oxide growth. ${ }^{13}$ The $\mathrm{CeO}_{2}$ films possess similar crystallographic texturing on the two different substrates. Hence, the respective $\mathrm{Ni} / \mathrm{CeO}_{2}$ surface and interfacial energies should be similar, which should lead to similar Ni particle size and shape. However, Fig. 7 clearly shows a striking difference exists. This difference in Ni size and shape (Fig. 7) may be attributed to the surface roughness of the $\mathrm{CeO}_{2}$ buffer layers and the respective $\mathrm{Ni} / \mathrm{CeO}_{2}$ interface. The $\mathrm{CeO}_{2}$ buffer layers are highly crystalline on both substrates. However, on $\mathrm{Si}$ the presence of native oxide and interfacial cerium silicate layers result in $\mathrm{CeO}_{2}$ layers with higher surface roughness values. The surface roughness affects the mobility of the Ni atoms impinging on the $\mathrm{CeO}_{2}$ layers.

Upon nucleation, Ni clusters form and their size is increased by capturing other $\mathrm{Ni}$ atoms either by diffusional processes or directly from the incident vapor. The Ni nanoparticle size and shape is governed by the number of $\mathrm{Ni}$ atoms that can be added to the cluster, which is affected by the characteristics of the $\mathrm{CeO}_{2}$ surface (surface energy and roughness) and the deposition time. Thurtell and Thurtell ${ }^{25}$ determined that diffusing species on rough surfaces scattered by asperities have decreased atomic velocities or mobilities. In our case, the Ni clusters become trapped in "valleys" along the $\mathrm{CeO}_{2}$ surface. The reduced mobility of the growing cluster results in the bigger, disk shaped $\mathrm{Ni}$ particles that we observed on the Si substrate [Fig. 7(a)]. On sapphire, the substrate/buffer layer interface is abrupt resulting in smooth $\mathrm{CeO}_{2}$ films. Atoms diffusing across the smooth surface are not as effectively scattered, hence this additional diffusion barrier present on rough surfaces does not exist. The amorphous regions at the $\mathrm{CeO}_{2} / \mathrm{Ni}$ interface on sapphire also play a role in tailoring the $\mathrm{Ni}$ particle shape. An amorphous interface favors a particle with spherical shape whereas crystalline templates usually lead to faceted islands because of surface energy anisotropy. ${ }^{18}$ According to STEM analysis [Fig. $7(b)$, the Ni particles in contact with amorphous $\mathrm{CeO}_{2}$ have spherical shapes with a narrow size distribution on sapphire. 
The observed narrow size distribution can be attributed to dipolar island edge-edge interactions, which induces a stable island size against coarsening. ${ }^{26}$

\section{SUMMARY AND CONCLUSIONS}

We studied the effect of substrate and thin film matrix on the magnetic properties of embedded self-assembled $\mathrm{Ni}$ nanoparticles. The nanoparticle/thin film composites were embedded into $\mathrm{Al}_{2} \mathrm{O}_{3}$, TiN, and $\mathrm{CeO}_{2}$ matrices on both $\mathrm{Si}$ and sapphire substrates by alternate ablation of respective targets during PLD. The ablation time on the Ni target was kept constant for all samples. Detailed structural (XRD and STEM) and magnetic characterization was performed. The magnetic properties vary widely depending on the matrix substrate combination. The $\mathrm{Ni}$ nanoparticles in $\mathrm{Al}_{2} \mathrm{O}_{3}$ and TiN were superparamagnetism and ferromagnetic in $\mathrm{CeO}_{2}$ on both $\mathrm{Si}$ and sapphire. Generally, $T_{B}$ and $H_{c}$ were enhanced with increased crystallinity of the thin film matrix. For crystalline $\mathrm{CeO}_{2}$ matrix layers, the $\mathrm{Ni}$ nanoparticles size and shape differed on the two substrates. On $\mathrm{Si}$, the particles were larger and disk shaped with larger interparticle separation $(>5 \mathrm{~nm})$. On sapphire, the $\mathrm{Ni}$ particles were roughly spherical and smaller with smaller particle-particle separation (1-2 $\mathrm{nm})$. The tighter particle packing on the sapphire substrate resulted in enhanced magnetic interactions between the Ni particles, reducing the coercivity at lower temperatures.

\section{ACKNOWLEDGMENTS}

This research was support by the following grants from the National Science Foundation NSF-NIRT 4-46109 (NCAT) and NSF-DMR 0213985 (MINT-UA). The research at ORNL was sponsored by the Division of Materials Science and Engineering of the US Department of Energy.

${ }^{1}$ B. D. Terris and T. Thomson, J. Phys. D 38, R199 (2005).

${ }^{2}$ T. Schrefl, G. Hrkac, D. Suess, W. Scholz, and J. Fidler, J. Appl. Phys. 93,
7041 (2003).

${ }^{3}$ C. Ross, Annu. Rev. Mater. Res. 31, 203 (2001).

${ }^{4}$ V. Skumryev, S. Stoyanov, Y. Zhang, G. Hadjipanayis, D. Girord, and J. Nogues, Nature (London) 423, 850 (2003).

${ }^{5} \mathrm{~B}$. D. Cullity, Introduction to Magnetic Materials (Addison-Wesley, Reading, MA, 1972).

${ }^{6}$ S. Kang, J. W. Harrell, and D. E. Nikles, Nano Lett. 2, 1033 (2002),

${ }^{7}$ Z. R. Dai, S. Sun, and Z. L. Wang, Nano Lett. 1, 443 (2001).

${ }^{8}$ Z. Gai, J. Y. Howe, J. Guo, D. A. Blom, E. W. Plummer, and J. Shen, Appl. Phys. Lett. 86, 023107 (2005).

${ }^{9}$ J.-M. Qiu and J.-P. Wang, Appl. Phys. Lett. 88, 192505 (2006), J.-M. Qiu, J. Bai, and J.-P. Wang, ibid. 89, 222506 (2006).

${ }^{10}$ C. Binns, K. N. Trohidou, J. Bansmann, S. H. Baker, J. A. Blackman, J.-P. Bucher, D. Kechrakos A. KIeibert, S. Louch, K.-H. Meiwes-Broer, G. M. Pastor, A. Perez, and Y. Xie, J. Phys. D 38, R357 (2005).

${ }^{11}$ N. B. Herndon, S.-H. Oh, J. T. Abiade, D. Pai, J. Sankar, S. J. Pennycook, and D. Kumar, J. Appl. Phys. 103, 07D515 (2008); D. Kumar, J. Narayan, A. V. Kvit, A. K. Sharma, and J. Sankar, J. Magn. Magn. Mater. 232, 161 (2001).

${ }^{12}$ D. Kumar, H. Zhou, T. K. Nath, A. V. Kvit, and J. Narayan, Appl. Phys. Lett. 79, 2817 (2001).

${ }^{13}$ C. Campbell, Surf. Sci. Rep. 27, 1 (1997).

${ }^{14}$ C. Jin, H. Zhou, W. Wei, and R. Narayan, Appl. Phys. Lett. 89, 261103 (2006).

${ }^{15}$ J. Kurian and M. Naito, Physica C 402, 31 (2004), X. Castel, M. Guillox-

Viry, A. Perrin, J. Lesueur, and F. Lalu, J. Cryst. Growth 187, 211 (1998).

${ }^{16}$ D. P. Norton, C. Park, J. D. Budai, S. J. Pennycook, and C. Prouteau, Appl. Phys. Lett. 74, 2134 (1999), J. Kang, X. Liu, G. Lian, Z. Zhang, G. Xiong, X. Guan, R. Han, and Y. Wang, Microelectron. Eng. 56, 191 (2001).

${ }^{17}$ T. Zheleva, K. Jagannadham, and J. Narayan, J. Appl. Phys. 75, 860 (1994), J. Narayan and B. C. Larson, ibid. 93, 278 (2003).

${ }^{18}$ H. Zhou, D. Kumar, A. Kvit, A. Tiwari, and J. Narayan, J. Appl. Phys. 94, 4841 (2003).

${ }^{19}$ G. Xiao and C. L. Chien, Appl. Phys. Lett. 51, 1280 (1987), G. Xiao and C. L. Chien, J. Appl. Phys. 63, 4252 (1988).

${ }^{20}$ A. Ceylan, C. C. Baker, S. K. Hasanain, and S. I. Shah, Phys. Rev. B 72, 134411 (2005).

${ }^{21}$ C. de Julián Fernández, Phys. Rev. B 72, 054438 (2005).

${ }^{22}$ B. Bian, D. Laughlin, K. Sato, and Y. Hirotsu, J. Appl. Phys. 87, 6962 (2000).

${ }^{23}$ Interplanar spacing $\left(d_{h k l}\right)=a_{0} /\left(h^{2}+k^{2}+l^{2}\right)^{1 / 2}$, where Ni lattice constant $a_{0}$ $=0.3524 \mathrm{~nm}$.

${ }^{24}$ N. V. Skorodumova, M. Baudin, and K. Hermansson, Phys. Rev. B 69, 075401 (2004).

${ }^{25}$ J. Thurtell and G. W. Thurtell, J. Chem. Phys. 88, 6641 (1988).

${ }^{26}$ F. Liu, Phys. Rev. Lett. 89, 246105 (2002). 